\title{
STATISTICAL Distributions OF Discrete WALSH HADAMARD TRANSFORM COEFFICIENTS OF NATURAL IMAGES
}

\author{
Vijay Kumar Nath and Deepika Hazarika \\ Department of Electronics and Communication Engineering, School of Engineering, \\ Tezpur University (A Central University), Napaam, Tezpur, Assam, India. \\ vknath@tezu.ernet.in and deepika@tezu.ernet.in
}

\begin{abstract}
For low bit rate applications, the discrete Walsh Hadamard transform (WHT) shows almost comparable results when compared to the popular discrete Cosine transform (DCT) in terms of compression efficiency, peak signal to noise ratio (PSNR) and visual results. The discrete WHT is a best choice which compromises between the computational complexity and compression efficiency. The great advantage of the discrete WHT is its relatively very low computational complexity when compared to DCT. However there is no definitive study reported in literature regarding the statistical distributions of discrete WHT coefficients of natural images. This study performs a $\chi^{2}$ goodness of fit test to determine the distribution that best fits the discrete WHT coefficients. The simulation results show that the distribution of a majority of the significant AC coefficients can be modelled by the Generalized Gaussian distribution. The knowledge of the appropriate distribution helps in design of optimal quantizers that may lead to minimum distortion and hence achieve optimal coding efficiency.
\end{abstract}

\section{KEYWORDS}

Image Compression, Discrete WHT, $\chi^{2}$ test, Statistical analysis

\section{INTRODUCTION}

DCT [1] has been very popularly used in many image and video coding standards like JPEG, MPEG1, MPEG2, H.261 [2,3]. But the computational complexity of the DCT is relatively very high which may not be preferred in several real time applications.

The discrete WHT $[4,5,6,18]$ is a orthogonal non sinusoidal transform which shows almost comparable performance in image compression applications when compared to DCT. The discrete WHT is popular for its very low computational complexity. The discrete WHT is considered as the best option whenever there is a compromise between the energy compaction capability and computational complexity. However till now, no study has been reported in the literature, on the distribution of discrete WHT coefficients of the natural images. In contrast, there is a huge amount of studies reported in the literature dealing with the distributions of 2D DCT coefficients of natural images $[7,8,9,10]$.

In [7], considering Gaussian, Laplacian, Gamma and Rayleigh distributions as probable models, Reininger and Gibson used Kolomogrov-Smirnov (KS) test and shown that DC coefficients can be well approximated by Gaussian distribution and AC coefficients can be well approximated by Laplacian distribution. In [8], the authors concluded that no particular density function can be used for each of the coefficients but Laplacian fits majority of the coefficients and when all 
the coefficients are lumped into one density function, the Cauchy distribution provides the best fit. In [9], Muller found that the Generalized Gaussian distribution best approximates the statistics of the 2D DCT coefficients. Smoot and Reeve [19] study the statistics of the DCT coefficients of the differential signal obtained after motion estimation.

They observe that the statistics are best approximated by the Laplacian distribution.

In this paper, considering Gaussian, Laplacian, Gamma, Generalized Gaussian and Cauchy distributions as probable models $[11,12]$, we study the statistical distribution using $\chi^{2}$ Goodness of Fit test [13]. The model distribution that gives the minimum $\chi^{2}$ statistic is chosen as the best fit. The $\chi^{2}$ test of fit showed that Generalized Gaussian distribution best models the statistics of discrete WHT coefficients of natural images. These results can be used to design optimal quantizers for discrete WHT coefficients. The organization of the paper is as follows. Section 2 provides the introduction to discrete WHT. $\chi^{2}$ Goodness of Fit test is explained in Section 3. Section 4 describes the model statistical distributions. Experimental results are presented in Section 5 and the paper is concluded in Section 6.

\section{DISCRETE WALSH HADAMARD TRANSFORM}

The basis functions of discrete WHT [4] are not sinusoids unlike Fourier and Cosine transforms. The basis functions are based on rectangular waveforms with peaks of \pm 1 . The N-point 1D discrete Walsh Hadamard transform of a signal $x(n)$ is given as

$$
V(z)=\frac{1}{N} \sum_{m=0}^{N-1} x(m) y_{z}(m), \quad z=0,1, \ldots \ldots \ldots \ldots \ldots, N-1
$$

where $y_{z}(m)$ is the Walsh function of order $z$ and is defined recursively as:

$$
\begin{aligned}
& y_{z}(m)=0 \text { for } m<0 \text { and } m>N-1 \\
& y_{0}(m)=1 \text { for } m=0,1 \ldots \ldots, N-1 \\
& y_{2 z}(m)=y_{z}(2 m)+(-1)^{z} y_{z}(2 m-1) \\
& y_{2 z+1}(m)=y_{z}(2 m)-(-1)^{z} y_{z}(2 m-1)
\end{aligned}
$$

for $z=0,1 \ldots \ldots \ldots . . . N-1$.

\section{3. $\chi^{2}$ GOODNESS OF FIT TEST}

$\chi^{2}$ and KS test [13] are the widely used goodness of fit test. The KS goodness of fit test statistic is a distance measure between the empirical cumulative distribution function (CDF) for a given data set and the given model cumulative distribution function. In contrast to the KS test, the $\chi^{2}$ goodness of fit test compares the model probability density functions with the empirical data and finds out the distortion by the following equation

$$
\chi^{2}=\sum_{i=1}^{k_{e}} \frac{\left(O_{i}-E_{i}\right)^{2}}{E_{i}}
$$

where the range of data is partitioned into $k_{e}$ disjoint and exhaustive bins $B_{i}, i=1,2,3, \ldots \ldots \ldots, k_{e}$. $E_{i}=n_{c} p_{i}$ is the expected frequency in bin $B_{i}$ where $p_{i}=P\left(x \in B_{i}\right)$ and $O_{i}$ is the observed frequency in bin $B_{i} . n_{c}$ is the total number of data samples. The model probability density function which gives the minimum $\chi^{2}$ value can be considered as the best fit.

The $\chi^{2}$ test is preferred over the KS test because for source coding the deviation from an assumed probability density function is more interesting than deviation from a distribution function [9]. 


\section{PROBABILITY DISTRIBUTIONS}

We use $\chi^{2}$ goodness of fit tests considering Gaussian, Laplacian, Gamma, Cauchy and Generalized Gaussian distributions [11,12] as probable models as these distributions are commonly used for statistical modeling of DCT coefficients. The parameters of the distributions were found using the maximum likelihood (ML) method.

\subsection{Gaussian Probability Density Function}

The Gaussian probability density function is given by

$$
f_{X}(x)=\frac{1}{\sqrt{2 \pi} \sigma} \exp \left(-\frac{(x-\mu)^{2}}{2 \sigma^{2}}\right)
$$

where $\mu$ is the mean and $\sigma^{2}$ is the variance. The ML estimates of $\mu$ and $\sigma^{2}$ are given by

$$
\begin{gathered}
\mu=\frac{1}{n} \sum_{i=1}^{n} x_{i} \\
\sigma^{2}=\frac{1}{n} \sum_{i=1}^{n}\left(x_{i}-\mu\right)^{2}
\end{gathered}
$$

\subsection{Laplacian Probability Density Function}

The Laplacian probability density function is given by

$$
f_{X}(x)=\frac{1}{2 a} \exp \left(-\frac{|x-\mu|}{a}\right)
$$

where $\mu$ is the mean and $a$ is the scale parameter. The variance is given by $2 a^{2}$. The ML estimate of the parameter $a$ is given by

$$
\hat{a}=\frac{1}{N} \sum_{i=1}^{N}\left|x_{i}-\not \mu\right|
$$

where $\mu$ is estimated using (4).

\subsection{Gamma Probability Density Function}

The Gamma probability density function is given by [14]

$$
f_{X}(x)=\frac{\sqrt[4]{3}}{\sqrt{8 \pi \sigma|x-\mu|}} \exp \left(-\frac{\sqrt{3}|x-\mu|}{2 \sigma}\right)
$$

The parameters $\mu$ and $\sigma$ are estimated using (4) and (5) respectively.

\subsection{Generalized Gaussian Probability Density Function}

The Generalized Gaussian probability density function $[11,12,15]$ is given by

$$
f_{X}(x)=\frac{\beta}{2 \alpha \Gamma\left(\frac{1}{\beta}\right)} \exp \left(-\frac{|x|}{\alpha}\right)^{\beta}
$$

where $\Gamma($.$) is the Gamma function given by$

$$
\Gamma(z)=\int_{0}^{\infty} e^{-t} t^{z-1} d t, z>0
$$

and $\alpha, \beta$ respectively are known as the scale parameter and the shape parameter. For the special cases $\beta=2$ or $\beta=1$, the generalized Gaussian pdf becomes a Gaussian or a Laplacian pdf respectively. The ML estimation of the parameters $\alpha$ and $\beta$ can be obtained as follows: 
The shape parameter $\beta$ is the solution of the equation

$$
1+\frac{\psi\left(\frac{1}{\beta}\right)}{\beta}-\frac{\sum_{i=1}^{N}\left|x_{i}\right|^{\beta} \log \left|x_{i}\right|}{\sum_{i=1}^{N}\left|x_{i}\right|^{\beta}}+\frac{\log \left(\frac{\beta}{N} \sum_{i=1}^{N}\left|x_{i}\right|^{\beta}\right)}{\beta}=0
$$

where $\psi($.$) is the digamma function given by$

$$
\psi(z)=\frac{\Gamma^{\prime}(z)}{\Gamma(z)}
$$

The ML estimate of $\alpha$ (for known ML estimate $\beta$ ) is given by

$$
\alpha=\left(\frac{\beta}{N} \sum_{i=1}^{N}\left|x_{i}\right|^{\beta}\right)^{\frac{1}{\beta}}
$$

where $N$ is number of observations.

The $\beta$ is determined using the Newton Raphson iterative procedure with the initial guess from the moment based method described in [16].

\subsection{Cauchy Probability Density Function}

The Cauchy probability density function $[11,12,17]$ is given by

$$
f_{X}(x)=\frac{1}{\pi}\left[\frac{\gamma}{\left(x-x_{0}\right)^{2}+\gamma^{2}}\right]
$$

where $x_{0}$ is the location parameter and $\gamma$ is the scale parameter. For the Cauchy distribution with sample size $N$, the likelihood function $L\left(x_{1}, \ldots \ldots . . x_{N} ; x_{0}, \gamma\right)$ is given by

$$
L\left(x_{1}, \ldots \ldots x_{N} ; x_{0}, \gamma\right)=\prod_{i=1}^{N}\left[\frac{1}{\pi \gamma\left\{1+\left(x_{i}-x_{0} / \gamma\right)^{2}\right\}}\right]
$$

The logarithm of the likelihood is given by

$$
\log L=-N \log \pi-N \log \gamma-\sum_{i=1}^{N} \log \left\{1+\left(\frac{x_{i}-x_{0}}{\gamma}\right)^{2}\right\}
$$

Maximizing the log likelihood function with respect to $y_{0}$ and $\gamma$ gives:

$$
\begin{gathered}
\sum_{i=1}^{N}\left(\frac{x_{i}-x_{0}}{\gamma}\right) /\left\{1+\left(\frac{x_{i}-x_{0}}{\gamma}\right)^{2}\right\}=0 \\
\sum_{i=1}^{N}\left\{1+\left(\frac{x_{i}-x_{0}}{\gamma}\right)^{2}\right\}^{-1}-\frac{N}{2}=0
\end{gathered}
$$

We use Newton Raphson iterative procedure to solve (15) and (16) for $x_{0}$ and $\gamma$. The median is used as an initial value for $x_{0}$ as described in [17].

\section{EXPERIMENTAL RESULTS}

The $\chi^{2}$ goodness of fit performance was evaluated using discrete WHT coefficients ( $8 \mathrm{x} 8$ block size) for fifteen natural test images having different characteristics but here we report only for popular Lena, Barbara, Aerial, House, Boats and Mandrill image. The parameters of all distributions were found using maximum likelihood method. The model distribution which 
Signal \& Image Processing : An International Journal (SIPIJ) Vol.3, No.1, February 2012

provides the minimum $\chi^{2}$ statistic is considered to be the best fit under the $\chi^{2}$ criterion. The nine AC coefficients $C_{10}, C_{11}, C_{01}, C_{20}, C_{02}, C_{12}, C_{21}, C_{03}$ and $C_{30}$ used in the experiments were chosen because they usually have the most effect on the image quality.

Table 1. $\chi^{2}$ statistics for a few discrete WHT coefficients of Lena (512x512) image

\begin{tabular}{|c|c|c|c|c|c|}
\hline Coefficient & Gaussian & Laplacian & Gamma & Gen. Gaussian & Cauchy \\
\hline$C_{10}$ & $5.94 \times 10^{8}$ & 8165 & 87 & $\mathbf{3 2}$ & 115 \\
\hline$C_{11}$ & $4.36 \times 10^{8}$ & 5740 & 103 & $\mathbf{2 2}$ & 205 \\
\hline$C_{01}$ & $4.61 \times 10^{7}$ & 954 & 60 & $\mathbf{4 3}$ & 296 \\
\hline$C_{20}$ & $4.23 \times 10^{7}$ & 2216 & 55 & $\mathbf{4 5}$ & 130 \\
\hline$C_{02}$ & $1.02 \times 10^{6}$ & 639 & $\mathbf{3 6}$ & 106 & 333 \\
\hline$C_{12}$ & $1.25 \times 10^{6}$ & 1071 & 42 & $\mathbf{2 7}$ & 134 \\
\hline$C_{21}$ & $1.42 \times 10^{6}$ & 1156 & $\mathbf{2 5}$ & 45 & 176 \\
\hline$C_{03}$ & $6.38 \times 10^{6}$ & 4292 & 168 & $\mathbf{8 8}$ & 163 \\
\hline$C_{30}$ & $1.61 \times 10^{12}$ & 20136 & 141 & $\mathbf{4 2}$ & 88 \\
\hline
\end{tabular}

Table 2. $\chi^{2}$ statistics for a few discrete WHT coefficients of Barbara (512x512) image

\begin{tabular}{|c|c|c|c|c|c|}
\hline Coefficient & Gaussian & Laplacian & Gamma & Gen. Gaussian & Cauchy \\
\hline$C_{10}$ & $1.00 \times 10^{10}$ & 3184 & 49 & $\mathbf{3 9}$ & 126 \\
\hline$C_{11}$ & $1.12 \times 10^{9}$ & 2060 & $\mathbf{1 4}$ & 18 & 162 \\
\hline$C_{01}$ & $1.47 \times 10^{4}$ & 365 & 35 & $\mathbf{2 2}$ & 274 \\
\hline$C_{20}$ & $5.48 \times 10^{4}$ & 200 & 122 & $\mathbf{2 1}$ & 196 \\
\hline$C_{02}$ & $1.53 \times 10^{8}$ & 380 & 30 & $\mathbf{9}$ & 197 \\
\hline$C_{12}$ & $1.67 \times 10^{6}$ & 1811 & 28 & $\mathbf{2 6}$ & 183 \\
\hline$C_{21}$ & $2.04 \times 10^{7}$ & 223 & 69 & $\mathbf{1 9}$ & 245 \\
\hline$C_{03}$ & $1.00 \times 10^{5}$ & 543 & $\mathbf{1 9}$ & 64 & 242 \\
\hline$C_{30}$ & $2.59 \times 10^{5}$ & 16608 & 34 & $\mathbf{8}$ & 147 \\
\hline
\end{tabular}

Table 3. $\chi^{2}$ statistics for a few discrete WHT coefficients of Aerial (256x256) image

\begin{tabular}{|c|c|c|c|c|c|}
\hline Coefficient & Gaussian & Laplacian & Gamma & Gen. Gaussian & Cauchy \\
\hline$C_{10}$ & 144 & 15 & 120 & $\mathbf{1 4}$ & 124 \\
\hline$C_{11}$ & 51 & 18 & 204 & $\mathbf{1 2}$ & 151 \\
\hline$C_{01}$ & 243 & 19 & 72 & $\mathbf{1 0}$ & 89 \\
\hline$C_{20}$ & 145 & 7 & 148 & $\mathbf{6}$ & 124 \\
\hline$C_{02}$ & 76 & 11 & 130 & $\mathbf{9}$ & 129 \\
\hline$C_{12}$ & 198 & 8 & 175 & $\mathbf{6}$ & 134 \\
\hline$C_{21}$ & 72 & 7 & 193 & $\mathbf{3}$ & 133 \\
\hline$C_{03}$ & 5726 & 28 & 35 & $\mathbf{7}$ & 79 \\
\hline$C_{30}$ & 286 & 42 & 79 & $\mathbf{2 7}$ & 99 \\
\hline
\end{tabular}


Signal \& Image Processing : An International Journal (SIPIJ) Vol.3, No.1, February 2012

Table 4. $\chi^{2}$ statistics for a few discrete WHT coefficients of House (256x256) image

\begin{tabular}{|c|c|c|c|c|c|}
\hline Coefficient & Gaussian & Laplacian & Gamma & Gen. Gaussian & Cauchy \\
\hline$C_{10}$ & 45678 & 1138 & 76 & $\mathbf{3 8}$ & 214 \\
\hline$C_{11}$ & $5.69 \times 10^{7}$ & 29566 & 101 & $\mathbf{2 0}$ & 40 \\
\hline$C_{01}$ & $1.42 \times 10^{5}$ & 1302 & 124 & $\mathbf{1 1 2}$ & 167 \\
\hline$C_{20}$ & 4903 & 952 & 152 & $\mathbf{1 1 8}$ & 169 \\
\hline$C_{02}$ & $8.18 \times 10^{6}$ & 2331 & 101 & 118 & $\mathbf{4 8}$ \\
\hline$C_{12}$ & $9.28 \times 10^{5}$ & 3758 & 51 & $\mathbf{1 3}$ & 26 \\
\hline$C_{21}$ & $1.34 \times 10^{6}$ & 688 & 31 & $\mathbf{1 6}$ & 68 \\
\hline$C_{03}$ & $1.81 \times 10^{13}$ & $2.33 \times 10^{7}$ & 295 & 83 & $\mathbf{3 3}$ \\
\hline$C_{30}$ & 24159 & 1738 & 110 & $\mathbf{5 2}$ & 124 \\
\hline
\end{tabular}

Table 5. $\chi^{2}$ statistics for a few discrete WHT coefficients of Boats (512x512) image

\begin{tabular}{|c|c|c|c|c|c|}
\hline Coefficient & Gaussian & Laplacian & Gamma & Gen. Gaussian & Cauchy \\
\hline$C_{10}$ & $2.19 \times 10^{5}$ & 741 & 43 & $\mathbf{1 7}$ & 142 \\
\hline$C_{11}$ & $8.60 \times 10^{4}$ & 636 & 65 & $\mathbf{1 2}$ & 143 \\
\hline$C_{01}$ & $7.48 \times 10^{8}$ & 3289 & 47 & $\mathbf{3 4}$ & 140 \\
\hline$C_{20}$ & $2.68 \times 10^{8}$ & 384 & 48 & $\mathbf{2 5}$ & 202 \\
\hline$C_{02}$ & $1.39 \times 10^{10}$ & 1551 & 66 & $\mathbf{3 3}$ & 155 \\
\hline$C_{12}$ & $1.15 \times 10^{7}$ & 698 & 13 & $\mathbf{5}$ & 171 \\
\hline$C_{21}$ & $6.81 \times 10^{5}$ & 450 & $\mathbf{1 4}$ & 23 & 189 \\
\hline$C_{03}$ & $1.91 \times 10^{13}$ & $6.14 \times 10^{4}$ & 128 & $\mathbf{1 3}$ & 146 \\
\hline$C_{30}$ & 54037 & 432 & $\mathbf{2 1}$ & 36 & 312 \\
\hline
\end{tabular}

Table 6. $\chi^{2}$ statistics for a few discrete WHT coefficients of Mandrill (512x512) image

\begin{tabular}{|c|c|c|c|c|c|}
\hline Coefficient & Gaussian & Laplacian & Gamma & Gen. Gaussian & Cauchy \\
\hline$C_{10}$ & 1774 & $\mathbf{2 1}$ & 388 & 23 & 398 \\
\hline$C_{11}$ & 781 & 6 & 248 & $\mathbf{5}$ & 415 \\
\hline$C_{01}$ & 15427 & $\mathbf{3 5}$ & 573 & 40 & 462 \\
\hline$C_{20}$ & 843 & 72 & 110 & $\mathbf{4 3}$ & 330 \\
\hline$C_{02}$ & 12848 & $\mathbf{1 5}$ & 329 & 20 & 421 \\
\hline$C_{12}$ & $2.31 \times 10^{5}$ & 4 & 288 & $\mathbf{2}$ & 369 \\
\hline$C_{21}$ & 1317 & 43 & 234 & $\mathbf{2 4}$ & 364 \\
\hline$C_{03}$ & 23950 & 7 & 352 & $\mathbf{6}$ & 407 \\
\hline$C_{30}$ & 2925 & 79 & 163 & $\mathbf{1 8}$ & 311 \\
\hline
\end{tabular}

If $N \times M$ is the size of the image after computation of 2D discrete WHT, then each coefficient will have $\frac{N}{8} \times \frac{M}{8}$ values for the image to be used in KS and $\chi^{2}$ test. Table 1, 2, 3, 4, 5 and 6 show the $\chi^{2}$ statistics of discrete WHT coefficients for Lena, Barbara, Aerial, House, Boats and Mandrill images respectively. The result indicates that the $\chi^{2}$ test statistic is smallest for the 
Signal \& Image Processing : An International Journal (SIPIJ) Vol.3, No.1, February 2012

General Gaussian distribution for most of the tested coefficients of all the images. Fig. 1 shows the empirical pdf of the discrete WHT coefficients along with the fitted Gaussian, Laplacian, Gamma, Generalized Gaussian and Cauchy pdfs. From Fig. 1 as well as from the values of the $\chi^{2}$ statistic, it is clear that the Generalized Gaussian distribution provides a better fit to the empirical distribution then that achieved by the Gaussian, Laplacian, Gamma and Cauchy pdfs.

\section{CONCLUSION}

In this paper, we perform the $\chi^{2}$ goodness of fit tests to determine an appropriate statistical distribution that best models the discrete WHT coefficients of images. The simulation results indicate that no single distribution can be used to model the distributions of all AC coefficients for all natural images. However the distribution of a majority of the significant AC coefficients can be approximated by the Generalized Gaussian distribution. The knowledge of the statistical distribution of transform coefficients is very important in the design of optimal quantizer that may lead to minimum distortion and hence achieve optimal coding efficiency.

\section{REFERENCES}

[1] N. Ahmad, T. Natarajan, and K. Rao, "Discrete Cosine Transform," IEEE transactions on Computers, vol. C-23, no. 1, pp. 90-93, 1974.

[2] V. Bhaskaran and K. Konstantinides, Image and Video compression standards - Algorithms and architectures, Kluwer Academic Publishers, 1997.

[3] W. B. Pennebaker and J. L. Mitchell, JPEG still image data compression standard, New York: Van Nostrand Reinhold, 1992.

[4] A. K. Jain, Fundamental of Digital Image Processing. Englewoods Cliffs, NJ : Prentice Hall, 1989.

[5] R. Costantini, J. Bracamonte, G. Ramponi, J. Nagel, M. Ansorge and F. Pellandini, "A low complexity video coder based on discrete Walsh Hadamard transform", In EUPSICO 2000 : European signal processing conference, pages 1217-1220, 2000.

[6] A. Aung, Boon Poh Ng, C. T. Shwe, "A new transform for document image compression", in Proc. of $7^{\text {th }}$ International Conference on Information, Communications and Signal Processing, 2009.

[7] R. C. Reininger and J. D. Gibson, "Distributions of the two dimensional dct coefficients for images," IEEE Transactions on Communications, Vol. 31, No. 6, pp. 835-839, 1983.

[8] J. D. Eggerton and M. D. Srinath, "Statistical distributions of image DCT coefficients," Computer Electrical Engineering, vol. 12, pp. 137- 145, 1986.

[9] F. Muller, "Distribution shape of two dimensional DCT coefficients of natural images," Electronics Letters, vol. 29, no. 22, pp. 1935-1936, 1993.

[10] R. L. Joshi and T. R. Fischer, "Comparison of Generalized Gaussian and Laplacian modeling in DCT image coding,” IEEE Signal Processing Letters, vol. 2, no. 5, pp. 81-82, 1995.

[11] N. L. Johnson, S. Kotz, N. Balakrishnan: Continuous Univariate Distributions John Wiley and Sons, 1994, Vol.1, $1^{\text {st }}$ edition.

[12] N. L. Johnson, S. Kotz, N. Balakrishnan: Continuous Univariate Distributions John Wiley and Sons ,1994, Vol.2, $2^{\text {nd }}$ edition.

[13] V. K. Rohatgi and A. K. E. Saleh, An Introduction to Probability and Statistics, John Wiley and Sons, 2001.

[14] N. S. Jayant and P. Noll, Digital Coding of waveforms. Prentice Hall, 1984.

[15] S. Mallat, "A theory for Multiresolution signal decomposition: The wavelet representation," IEEE Transactions on Pattern Recognition Machine Intelligence, vol. 11, pp. 674-693, 1989.

[16] K. Sharifi and A. Leon-Garcia, "Estimation of shape parameter for Generalized Gaussian distributions in subband decompositions of video," IEEE Transactions on Circuits and Systems for Video Technology, vol. 5, no. 1, p. 5256, 1995.

[17] G. Haas, L. Bain and C. Antle, "Inferences for the Cauchy distribution based on maximum likelihood estimators. Biometrika, Vol. 57, No.2, pp. 403-408, 1970.

[18] A. N. Akansu and M. S. Kadur, "Discrete Walsh Hadamard transform vector quantization for motion compensated frame difference signal coding" International Conference on Accoustics, Speech and Signal Processing (ICASSP), UK, 1989. 
Signal \& Image Processing : An International Journal (SIPIJ) Vol.3, No.1, February 2012

[19] S. R. Smoot and L. A. Rowe, "Study of det coefficient distributions," SPIE, vol. 2657, pp. 403411, 1996.

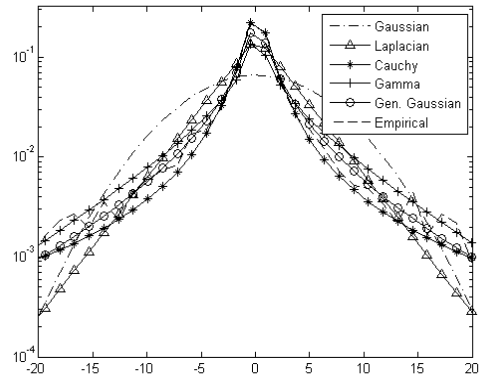

(a) $C_{10}$

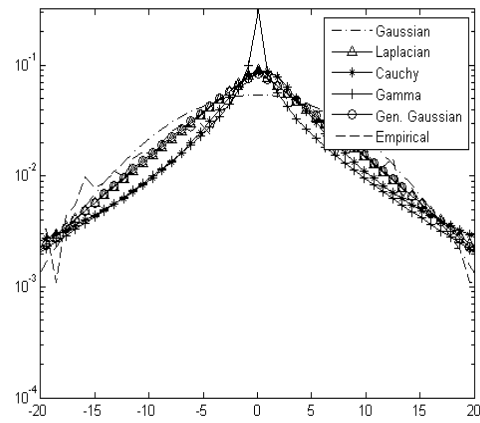

(c) $C_{11}$

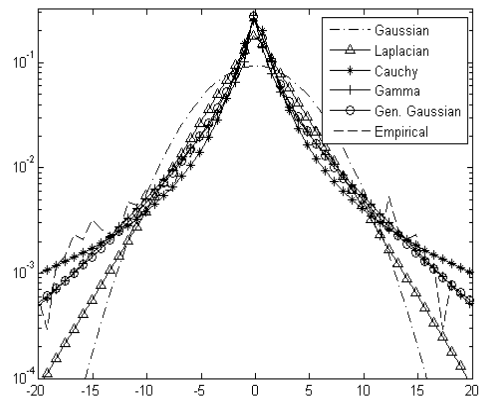

(e) $C_{20}$

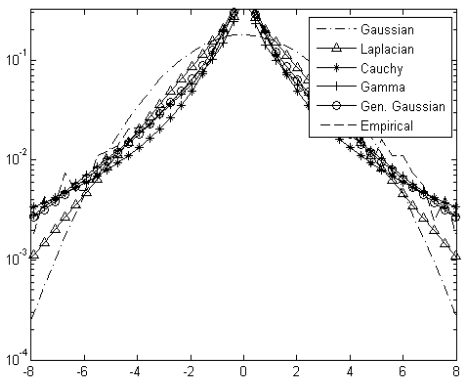

(b) $C_{21}$

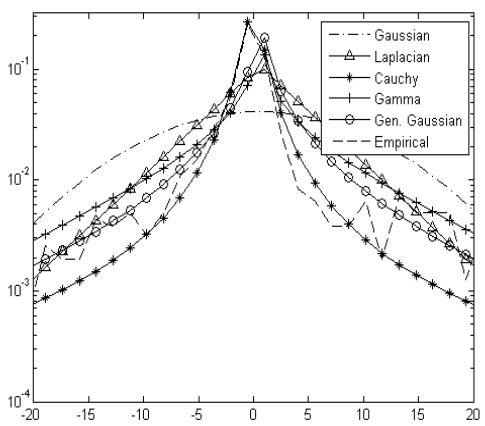

(d) $C_{10}$

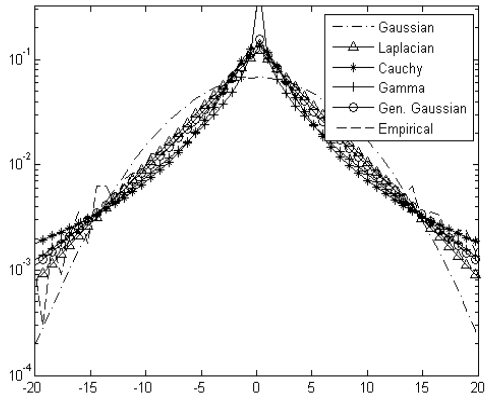

(f) $C_{20}$

Figure1. Logarithmic Histograms of the discrete WHT coefficients for (a) Lena (b) Barbara (c) Aerial (d) House (e) Boats (f) Mandrill images and the Gaussian, Laplacian, Gamma, Generalized Gaussian and Cauchy pdfs fitted to this histogram in log domain 